\title{
UNIVERSITY OF TOKYO \\ RADIOCARBON MEASUREMENTS II
}

\author{
JUN SATO,* TOMOKO SATO,** YASUKO OTOMORI,** \\ and HISASHI SUZUKI $\dagger$
}

Carbon Dating Laboratory, University of Tokyo, Tokyo, Japanł

Radiocarbon measurements in the list below were made during the period from Sept. 1967 to Aug. 1968. Our measuring system is based on acetylene counting in an Oeschger-Houtermans-type proportional counter $(1 \mathrm{~L})$ at a pressure of $1 \mathrm{~atm}$. All data are based on duplicated measurements. For calculation of ages, $95 \%$ activity of NBS oxalic acid is used as the modern standard and the value of $5570 \pm 30$ years is used for the half-life of $\mathrm{C}^{14}$. Details of procedures are given in the previous report (Radiocarbon, 1968, v. 10, p. 144-148).

We measure bone samples for carbon in two forms: $\mathrm{CO}_{2}$ released from bones by treatment with $20 \% \mathrm{H}_{2} \mathrm{SO}_{4}$ and organic material extracted in the treatment.

Descriptions of samples are given by collectors and submitters. We express our thanks to Miss Ayako Nakamura for her secretarial assistance.

\section{SAMPLE DESCRIPTIONS}

I. GEOLOGIC SAMPLES

Japan

\section{TK-14. Tsukitono-machi \\ $31,500 \pm 600$ \\ 29,500 в.c.}

Wood from gravel from upper terrace along Tone R., Tsukitonomachi, Gumma Pref. (36 $45^{\prime} \mathrm{N}$ Lat, $139^{\circ} 0^{\prime} \mathrm{E}$ Long). Coll. 1965 by $\mathrm{S}$. Iwatsuka, Dept. Geog., Univ. Tokyo; subm. 1966 by S. Iwatsuka.

TK-26. Senami

$<0.6 \%$ of modern

Calcite crystals precipitated from flowing hot spring of Ryûsenkaku well at Senami Spa $\left(38^{\circ} 14^{\prime}\right.$ N Lat, $139^{\circ} 27^{\prime}$ E Long), Niigata Pref. Coll. and subm. by N. Katayama, Univ. Tokyo.

\section{TK-29. Shimohara shell bed}

$8760 \pm 100$ 6810 B.c.

Shells from marine terrace sediments (Shimohara formation), depth $8 \mathrm{~m}$ (alt $16 \mathrm{~m}$ ), Haneo, Tachibana-cho, Kanagawa Pref. (35 $17^{\circ} 30^{\prime \prime} \mathrm{N}$ Lat, $139^{\circ} 14^{\prime} 10^{\prime \prime}$ E Long). Terrace (alt $26 \mathrm{~m}$ ) is highest Holocene marine terrace in Japan. Coll. 1967 by K. Endo et al., Univ. Tokyo; subm. 1967 by S. Iwatsuka. Comment (S.I.): another date on same sample, $7840 \pm$ 100 (GaK-1598). Dates coincide with other dates of early phase of maximum transgression of Holocene.

* Department of Chemistry, Faculty of Science, University of Tokyo

** Department of Anthropology, Faculty of Science, University of Tokyo

† Chairman, Carbon Dating Committee, University of Tokyo

Mailing address: c/o Department of Anthropology, Faculty of Science, University of Toyko, Bunkyo-ku, Tokyo, Japan 


\section{Haruna Volcano series}

\section{TK-31. First Pumice flow deposits}

Charred stem of wood from 1st pumice flow deposits on $\mathrm{S}$ foot of Haruna Volcano, Gumma Pref. (36 ${ }^{\circ} 22.5^{\prime} \mathrm{N}$ Lat, $138^{\circ} 54.2^{\prime} \mathrm{E}$ Long). Coll. 1965, 1967 by O. Oshima, Univ. Tokyo and subm. 1967 by F. Takai, Univ. Tokyo. Comment (F.T.): date consistent with $24,000 \pm 650$ (GaK-725, Radiocarbon, 1967, v. 9, p. 46) given for stratigraphically younger mudflow sediment.

\section{TK-32. Futatsu-dake Pumice fall}

$$
2530 \pm 90
$$

Charcoal from Futatsu-dake pumice fall on caldera rim, Haruna Volon summit of Coll. 1965 Coll. 1965 by O. Oshima; subm. 1967 by F. Takai. Comment (F.T.): archaeologic datum suggests pumice fall in 6th cent. A.D.

\section{Fuji Volcano series}

Carbonized wood from Fuji Volcano. Coll. 1964 by H. Tsuya; subm. 1967, 1968 by S. Aramaki, Earthquake Res. Inst., Univ. of Tokyo.

\section{TK-36. South slope}

$$
2840 \pm 80
$$

Carbonized wood fromed from summit crater, near trail from Fujino-miya, a very recent lava flow Lat, $138^{\circ} 45^{\prime}$ E Long).

\section{TK-37. Northwestern slope}

$$
1940 \pm 100
$$

Carbonized wood from wall of lava tree mold. Lava flow erupted from one of lateral vents at ca. $+2830 \mathrm{~m}$, N slope. Sample coll. at +1840 $\mathrm{m}\left(35^{\circ} 25^{\prime} \mathrm{N}\right.$ Lat, $138^{\circ} 40^{\prime} \mathrm{E}$ Long).

\section{TK-47. Western slope}

$$
1780 \pm 90
$$

\section{A.D. 120}

Carbonized wood from ash underlying recent lava flow, Nishimarubi, at Osawa parking area, automobile toll rd. $\left(35^{\circ} 22^{\prime} \mathrm{N}\right.$ Lat, $138^{\circ}$ 42' E Long).

\section{TK-38. Takeda River}

$24,900 \pm 450$

Peat from deposit of lower terraces along Takeda R., Tari, Kasugacho, Hyogo Pref. (35 $10^{\prime} \mathrm{N}$ Lat, $135^{\circ} 6^{\prime}$ E Long). Coll. 1967 by A. Okada, Dept. Geog., Univ. Tokyo; subm. 1967 by S. Iwatsuka. Comment (S.I.): plants and pollen analysis indicate that peat layer (Kotari peat layer) probably accumulated at climax of Würm Age.

TK-39. Ikeda

$27,700 \pm 600$

25,750 в.c.

Wood from terrace deposit dislocated by Ikeda fault (Median Dislocation Line) at Ikeda, Tokushima Pref. (34 $1^{\prime}$ N Lat, $133^{\circ} 48^{\prime}$ E Long). 
Coll. 1967 by A. Okada; subm. 1967 by S. Iwatsuka. Comment (S.I.): same sample gave 23,600 \pm 1000 (GaK-1614).

TK-45. Tsuchiya

Charcoal in pumice flow overlying Tsuchizawa formation at Tsuchiya, Hiratsuka city, Kanakawa Pref. (35 $21^{\prime} \mathrm{N}$ Lat, $139^{\circ} 15.5^{\prime}$ E Long). Coll. 1967 and subm. 1968 by N. Katayama.

\section{TK-46. Takao}

$30,800 \pm 800$

Fossil trunk in muck buried under pumice fall and flow effused from crater nearby, at cutting of Tômei Hwy, S of Takao, Ôi town, Kanagawa Pref $\left(35^{\circ} 20^{\prime} \mathrm{N}\right.$ Lat, $139^{\circ} 11^{\prime} \mathrm{E}$ Long). Coll. and subm. 1968 by $\mathrm{N}$. Katayama.

\section{TK-48. Oiso}

$3000 \pm 180$

Charcoal from black humic sand layer buried in dune sand, NishiKoiso, Oiso-cho, Kanagawa Pref. (35 $18^{\prime} \mathrm{N}$ Lat, $139^{\circ} 17^{\prime}$ E Long). Coll. 1968 by K. Endo; subm. 1968 by S. Iwatsuka. Comment (S.I.): result consistent with age of Later Jomon pottery in humic layer. Holocene sand dunes are divided by this layer into 2 layers formed before and after 3000 B.P.

\section{Yoshino River series}

TK-50. Nomura

$31,500 \pm 960$

29,550 в.c.

Wood from deposit of alluvial fans formed by tributaries of Yoshino R. at Nomura, Waki-machi, Mima-gun, Tokushima Pref. (34 $3^{\prime} 32^{\prime \prime} \mathrm{N}$ Lat, 134 5' 44" E Long). Coll. 1967 by A. Okada; subm. 1968 by S. Iwatsuka.

\section{TK-51. Sôjanomoto}

Peaty clay from fan gravel along tributary of Yoshino R. at Sôjanomoto, Ichiba-cho, Awa-gun, Tokushima Pref. (34 $5^{\prime} \mathrm{N}$ Lat, 134 $18^{\circ} 20^{\prime \prime}$ E Long). Coll. 1968 by A. Okada; subm. 1968 by S. Iwatsuka.

\section{ARCHAEOLOGIC SAMPLES}

\section{Amud Cave series}

\section{A. Israel}

Materials from Amud cave, Galilee Dist., Israel (32 $52^{\prime} 30^{\prime \prime} \mathrm{N}$ Lat, $35^{\circ} 30^{\prime} 09^{\prime \prime}$ E Long) which yielded Neanderthal skeletons.

\section{TK-12. Black soil}

Organic materials in black soil from base of Bed B of cave deposits, ca. $1 \mathrm{~m}$ lower than horizon of Neanderthal skeleton and artifacts. Coll. 1964 by Tokyo Univ. Scientific Expedition to W Asia and subm. 1966 by F. Takai. Comment (F.T.): bone carbonate in mammalian bones from same bed gave $10,500 \pm 140$ (TK-33a, this list). 
TK-33a. Animal bone

Bone carbonate in mammalian bones from middle horizon of Bed B of cave deposits, nearly same horizon to Neanderthal skeleton, assoc. with upper Levalloiso-Mousterian type stone artifacts. Coll. 1964 by Tokyo Univ. Scientific Expedition to $\mathrm{W}$ Asia and subm. 1967 by $\mathrm{F}$. Takai. Comment (F.T.): black soil from ca. $1 \mathrm{~m}$ lower horizon of same site gave $10,600 \pm 400$ (TK-12, this list).

\section{Konjiki-do series}

$$
\text { B. Japan }
$$

Building materials from Konjiki-do temple at Hiraizumi, Iwate Pref. $\left(29^{\circ} 00^{\prime} \mathrm{N}\right.$ Lat, $141^{\circ} 06^{\prime} \mathrm{E}$ Long) built in 1st yr of Tenji (A.D. 1124). Age confirmed by inscription on hidden ridge, Chuson-ji. Coll. and subm. 1967 by M. Sekino, Univ. Tokyo.

\section{TK-27. Wood}

$1200 \pm 80$

Wood (Thujopsis dolarbata Sieb. et Zucc. var. hondai Makino) with bark, from 1 of roof struts.

TK-28. Texture

Hemp (Cannabis Sativa L.) used as cloth foundation coated with lacquer on wooden wall.

\section{Nara Palace series}

Materials from pit in site of ancient Nara Palace, Nara Pref. (24. $40^{\prime}$ N Lat, $135^{\circ} 49^{\prime}$ E Long). Subm. 1967 by M. Sekino.

TK-40. Charcoal with hark

$2390 \pm 90$

TK-41. Bark of Japanese Cypress 440 B.C.

\section{(Chamaecyparis obtusa Endl.)}

$$
\begin{aligned}
& 2300 \pm 110 \\
& 350 \text { в.C. }
\end{aligned}
$$

Comment (M.S.): found with wooden clip with inscription of 19th yr of Tempyô (A.D. 747).

\section{TK-52. Gifu II}

Charcoal from Pit House No. W4 at Gifu II site, Tokoro-machi, Tokoro-gun, Hokkaido (44 $07^{\prime} \mathrm{N}$ Lat, $143^{\circ} 59^{\prime} \mathrm{E}$ Long). Excavation 1965 by T. Mikami, Dept. Archaeol., Univ. Tokyo. Pottery is of Satsumon type. Coll. 1965 and subm. 1968 by T. Saito, Univ. Tokyo. Comment (T.S.): compared with result of TK-21 (Radiocarbon, 1968, v. 10, p. 146), true age of sample is supposedly 1230 to 1300 A.D., which is reasonable for our archaeologic chronology. Cf. Gifu II site series, (Radiocarbon, 1968, v. 10, p. 147).

TK-53. Wakka site

Charcoal from Pit House No. 3 at Wakka site, Tokoro-machi, 
'Tokoro-gun, Hokkaido ( $44^{\circ} 08^{\prime} \mathrm{N}$ Lat, $143^{\circ} 54^{\prime} \mathrm{E}$ Long). Excavation 1967 by T. Saito; subm. 1968 by T. Saito. Pottery is of Satsumon type. Comment (T.S.): compared with result of TK-21 (Radiocarbon, 1968, v. 10 , p. 146), true age of sample is supposedly 1230 to 1300 A.D., which is reasonable for our archaeologic chronology.

TK-54. Sakaeura II site

$1240 \pm 90$

Charcoal from Pit House No. 7 at Sakaeura II site, Tokoro-machi, Tokoro-gun, Hokkaido $44^{\circ} 07^{\prime} \mathrm{N}$ Lat, $144^{\circ} 03^{\prime}$ E Long). Excavation 1967 by T. Saito; subm. 1968 by T. Saito. Pottery is of Okhotsk type. Comme'nt (T.S.): compared with result of TK-27 and 28 (this list), true age is supposedly 1100 to 1160 A.D. TK-2, 4, 9, and 17 (Radiocarbon, 1968, v. 10, p. 147) should be same age as TK-54.

\section{Telul eth-Thalathat II series}

\section{B. Iraq}

Telul eth-thalathat, early village sites, ca. $60 \mathrm{~km} \mathrm{~W}$ of Mosul, Iraq $\left(36^{\circ} 25^{\prime} \mathrm{N}\right.$ Lat, $42^{\circ} 37^{\prime} \mathrm{E}$ Long). Culture revealed from bottom 2 levels is so-called "Neolithic Hassuna." Pottery is quite similar to that of Tell Hassuna and Matarrah. Coll. 1964 by S. Miyake, Tokyo Univ. Iraq-Iran Archaeol. Expedition; subm. 1966 by N. Egami, Univ. Tokyo.

\section{TK-23. Level XVI}

$7360 \pm 100$ Carbonized grass (?) from hearth of Pit House No. 3.

TK-24. Level XV 5410 B.C.

Carbonized grass and charcoal (?) from black humus of Level XV.

$7520 \pm 120$ $\mathbf{5 5 7 0}$ в.C.

$4200 \pm 90$

TK-25. Telul eth-Thalathat $\mathrm{V}$, granary

2250 B.C.

Carbonized wheat from rectangular granary of site, ca. $60 \mathrm{~km} \mathrm{~W}$ of Mosul, Iraq ( $36^{\circ} 25^{\prime} \mathrm{N}$ Lat, $42^{\circ} 37^{\prime} \mathrm{E}$ Long). Pottery is of typical Ninevite 5. Coll. 1965 by Y. Chiyonobu, Tokyo Univ. Iraq-Iran Archaeol. Expedition; subm. 1966 by N. Egami.

\section{Tal-i-Mushki series}

$$
\text { C. Iran }
$$

Tal-i-Mushki, early village site, in Marv-Dasht, Fars, Iran $\left(29^{\circ} 50^{\prime}\right.$ $\mathrm{N}$ Lat, $52^{\circ} 45^{\prime} \mathrm{E}$ Long). Unique painted pottery and geometric microlith were found. Coll. 1965 by Y. Chiyonobu, Tokyo Univ.; subm. 1967 by S. Fukai, Univ. of Tokyo.

TK·34. Charcoal

From black humus of Level 2a.

TK-35a. Animal bone, carbonate
$8640 \pm 120$ 6690 в.c.

$3610 \pm 110$ 1660 в.c. 
TK-35b. Animal bone, organic

$6800 \pm 600$

From Level 2a.

4850 в.c.

\section{Peru}

TK-30. Batangrande, pre-Chavín phase

$2880 \pm 100$

930 в.C.

Charcoal from tombs at Batangrande, Prov. Ferrenafe, Dept. Lambayeque, Peru $\left(6^{\circ} 42^{\prime} \mathrm{S}\right.$ Lat, $80^{\circ} 12^{\prime} \mathrm{W}$ Long), assoc. with small stone implements and potsherds different from Chavín. Coll. 1967 by J. Zevallos, Archaeol. Soc. of Lambayeque; subm. 1967 by K. Terada, Univ. Tokyo.

\section{Kotosh preceramic temple series}

Charcoal from hearths of temples at Kotosh, Dept. Huánuco, Peru $\left(9^{\circ} 56^{\prime} \mathrm{S}\right.$ Lat, $76^{\circ} 16^{\prime} 30^{\prime \prime} \mathrm{W}$ Long). Coll. 1966 and subm. 1967 by $\mathrm{S}$. Izumi, Univ. Tokyo.

TK-42. Temple ER-26

$3900 \pm 900$ 1950 B.C.

Comment (S.I.): dates of other temples of same period at Kotosh are $3620 \pm 100(\mathrm{GaK}-766 \mathrm{a}), 3900 \pm 100(\mathrm{GaK}-776 \mathrm{~b})$ and $2040 \pm 100$ (GaK-764).

\section{TK-44. Temple ER-20}

Comment (S.I.): dates of same period are cited in TK-42. This date is almost parallel to $2040 \pm 100$ (GaK-764), but they are too late for preceramic period at site.

\section{TK-43. Shillacoto, Kotosh Wairajirca period}

$3200 \pm 80$ jir charcos Wairajirca period at Shillacoto site, Dept. Huánuco, Peru $\left(9^{\circ} 56^{\prime} \mathrm{S}\right.$ Lat, $76^{\circ} 16^{\prime}$ W Long). Coll. 1966 and subm. 1967 by S. Izumi. Comment (S.I.): dates of parallel period at Kotosh site are $3100 \pm 130$ (N-69-2, Radiocarbon, 1966, v. 8, p. 336), $3800 \pm 110$ (GaK-262) and $3780 \pm 90$ (GaK-765).

Date lists:

$\begin{array}{ll}\text { Gakushuin VI } & \text { Kigoshi, 1967 } \\ \text { Riken II } & \text { Yamasaki, Hamada, and Fujiyama, } 1966 \\ \text { Tokyo I } & \text { Sato, Sato, and Suzuki, 1968 }\end{array}$

Kigoshi, Kunihiko, 1967, Gakushuin natural radiocarbon measurements VI: Radiocarbon, v. 9, p. 43-62.

Sato, Jun, Sato, Tomoko, and Suzuki, Hisashi, 1968, University of Tokyo radiocarbon measurements I: Radiocarbon, v. 10, p. 144-148.

Yamasaki, Fumio, Hamada, Tatsuji, and Fujiyama, Chikako, 1966, Riken natural radiocarbon measurements II: Radiocerbon, v. 8, p. 324-339. 